\title{
Interplay of Chemistry and Faceting at Grain Boundaries in a Model Al Alloy
}

\author{
Huan Zhao, ${ }^{1, *}$ Liam Huber, ${ }^{1}$ Wenjun Lu, ${ }^{1, \dagger}$ Nicolas J. Peter, ${ }^{1}$ Dayong An, ${ }^{1}$ Frédéric De Geuser®${ }^{2}$ \\ Gerhard Dehm, ${ }^{1}$ Dirk Ponge, ${ }^{1}$ Jörg Neugebauer, ${ }^{1}$ Baptiste Gault ${ }^{1,3, \$}$ and Dierk Raabe ${ }^{1}$ \\ ${ }^{1}$ Max-Planck-Institut für Eisenforschung, Max-Planck-Straße 1, 40237 Düsseldorf, Germany \\ ${ }^{2}$ Université Grenoble Alpes, CNRS, Grenoble INP, SIMaP, F-38000 Grenoble, France \\ ${ }^{3}$ Department of Materials, Royal School of Mines, Imperial College London, London SW7 2AZ, United Kingdom
}

(Received 5 September 2019; accepted 2 January 2020; published 9 March 2020)

\begin{abstract}
The boundary between two crystal grains can decompose into arrays of facets with distinct crystallographic character. Faceting occurs to minimize the system's free energy, i.e., when the total interfacial energy of all facets is below that of the topologically shortest interface plane. In a model Al-Zn-Mg-Cu alloy, we show that faceting occurs at investigated grain boundaries and that the local chemistry is strongly correlated with the facet character. The self-consistent coevolution of facet structure and chemistry leads to the formation of periodic segregation patterns of 5-10 nm, or to preferential precipitation. This study shows that segregation-faceting interplay is not limited to bicrystals but exists in bulk engineering $\mathrm{Al}$ alloys and hence affects their performance.
\end{abstract}

DOI: 10.1103/PhysRevLett.124.106102

Grain boundaries (GBs) are regions connecting adjacent crystals with different crystallographic orientations. GBs are a type of lattice imperfection [1,2], with their own structure and composition, and as such impact a material's mechanical and functional properties [3]. Structural motifs and phases formed at chemically decorated GBs can be of a transient nature [4-7] or are local thermodynamic structural-chemical equilibrium states [8-11]. General GBs exhibit a wide range of local crystallographic structure and facet motifs. In-plane GB structural motifs arise naturally from topological and bonding constraints associated with the adjacent crystals. An initially flat GB can decompose into a series of sequentially arranged facets, driven by the reduction in total interfacial energy. This counterintuitive transition, where the total length of the faceted interface exceeds that of the shortest interface, originates from the interplay between the interfacial energy of individual facets and their crystallographic character. Altogether, the longer interface can be of overall lower free energy [12-15].

GBs often see the segregation of solutes or impurities, driven by a reduction of the interfacial energy according to the Gibbs adsorption isotherm, and are favorable sites for heterogeneous nucleation of precipitates. These can form during quenching [16] or during natural or artificial aging in $\mathrm{Al}$ alloys $[17,18]$. Diffusion, segregation, and precipitation are

Published by the American Physical Society under the terms of the Creative Commons Attribution 4.0 International license. Further distribution of this work must maintain attribution to the author(s) and the published article's title, journal citation, and DOI. Open access publication funded by the Max Planck Society. intimately related to the local GB structure, which can result in strain localization, intergranular fracture, and corrosion [19]. The interaction between GB composition and structure was mostly studied on well-defined bicrystals at microscopic $[5,14,20-22]$ and atomic scales $[11,12,23,24]$. However, such model samples can be far from representative when compared to bulk materials processed via conventional routes.

Here, we reveal the interplay between composition and structure at GBs in an engineering material by using aberration-corrected scanning transmission electron microscopy (STEM), atom probe tomography (APT) and atomistic simulations. Near $\Sigma 5, \Sigma 11$, and $\Sigma 13 a$ coincidentsite lattice boundaries were studied in a coarse-grained Al-6.22\%Zn-2.46\% Mg-2.13\% Cu alloy (wt. \%). These boundaries were selected because specimens for highresolution STEM require that both grains are along a common low-index zone axis to image individual atomic columns. The cast material was homogenized, hot rolled, and then solution heat treated at $475^{\circ} \mathrm{C}$, followed by water quenching. Details are in the Supplemental Material [25]. The investigated GBs decompose into facets ranging in length from micrometers down to only a few nanometers. A distinct segregation and precipitation behavior is observed on the different facets. Our findings are of interest primarily for two reasons: first, little is known about the interplay of GB chemistry and faceting in general and in particular in engineering Al alloys; second, segregation and precipitation on specific GB facets influence strain localization, damage initiation and corrosion, and thereby materials' macroscopic properties.

The first investigated GB was a near $\Sigma 11$ GB from the as-quenched $\mathrm{Al}-\mathrm{Zn}-\mathrm{Mg}-\mathrm{Cu}$ alloy. APT and STEM results are presented in Fig. 1. The GB, aligned edge-on, is 

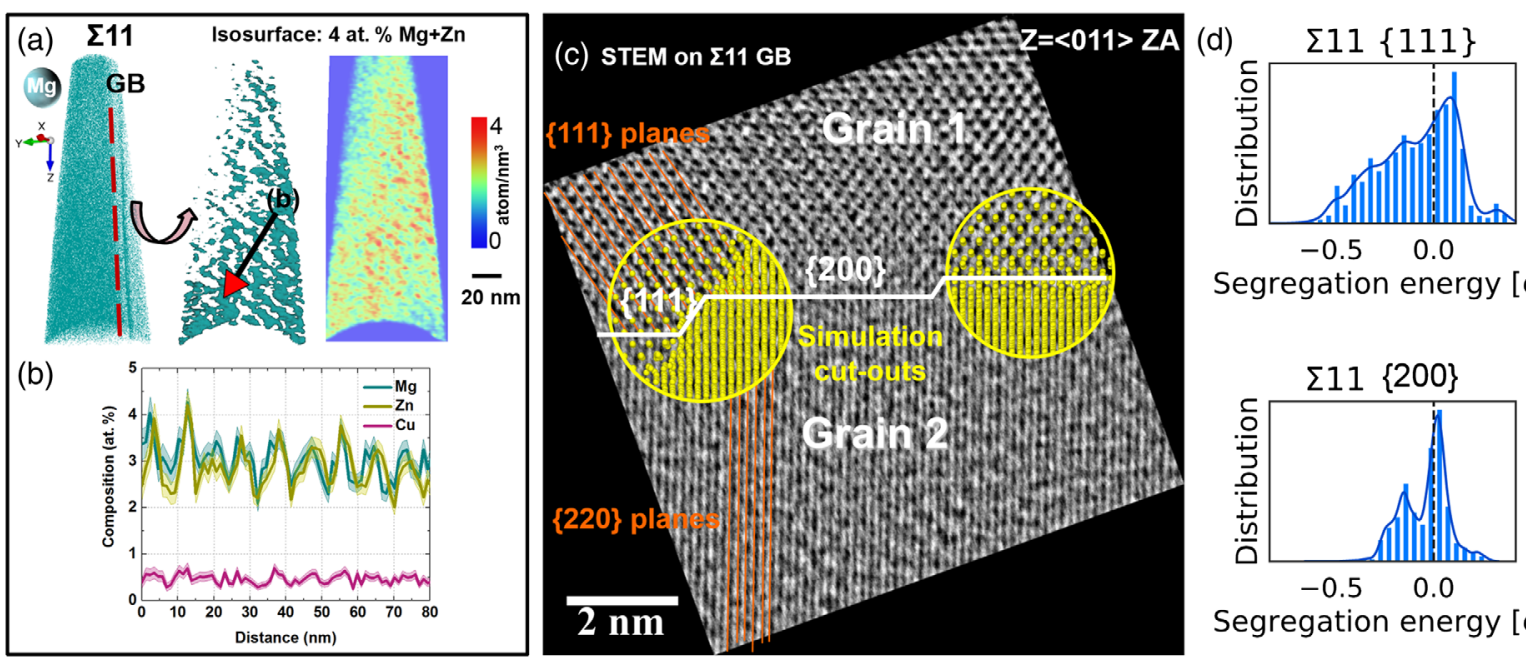

Segregation energy $[\mathrm{eV}]$

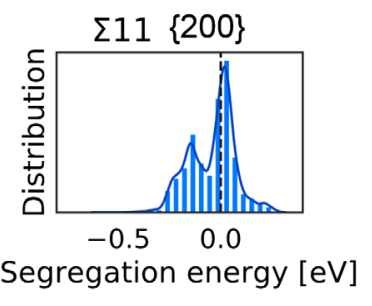

FIG. 1. (a) Atom map of Mg of a $\Sigma 11 \mathrm{~GB}$ in the as-quenched state along with the isocomposition surfaces and the projected density map of $\mathrm{Mg}$ and $\mathrm{Zn}$ within the GB plane (region indicated by the red line); (b) Corresponding composition profile along the arrow in (a); (c) BF STEM image of a $\Sigma 11\langle 011\rangle \mathrm{GB}$, which contains nanometer-sized facets (with inset atomic model) showing the atomic structure of the two GB facets; (d) Dilute segregation energy distributions for $\mathrm{Mg}$ at $\{111\}$ and $\{200\}$ planes of $\Sigma 11$ boundaries.

highlighted in the APT analysis in Fig. 1(a). It is already substantially enriched with solutes by the end of the quench. Figure 1(a) also shows a plane-view projection of the GB. The isocomposition surfaces of 4 at. $\% \mathrm{Mg}+\mathrm{Zn}$ and the two-dimensional density map show that $\mathrm{Mg}$ and $\mathrm{Zn}$ follow periodic segregation patterns across the GB plane, with a period of 6-10 $\mathrm{nm}$. The corresponding longitudinal composition profile is shown in Fig. 1(b), with the error bars representing the standard deviation of the counting statistics in each bin $(2 \sigma)$. It reveals a periodic distribution of $\mathrm{Mg}$ and $\mathrm{Zn}$. A $\Sigma 13 a$ GB shows a similar periodic segregation behavior with a $\sim 5 \mathrm{~nm}$ period (Supplemental Material, Fig. 1 [25]).

Figure 1(c) shows evidence of faceting at a $\Sigma 11 \mathrm{~GB}$ in the same sample, viewed by a bright field (BF) STEM close to a $\langle 011\rangle$ zone axis of the two grains. The average GB plane is close to $\{811\}$ determined from the upper grain. Being from a bulk alloy, both grains cannot be perfectly in zone, preventing the observation of atomic-scale motifs. Despite this limitation, two types of facets are identified: longer $\{200\}$ facets of approximately $4 \mathrm{~nm}$, and shorter $\{111\}$ facets of approximately $0.5-1 \mathrm{~nm}$. Alternating $\{200\}$ and $\{111\}$ type facets with a length ratio of $\sim 5$ coexist along the GB.

To better understand the interplay of the anisotropic segregation and the structural faceting of the GB, we performed computational simulations of $\mathrm{Mg}$ segregation energies to $\{200\}$ and $\{111\}$ facets of a similar $\Sigma 11$ boundary. We used the Large-Scale Atomic/Molecular Massively Parallel Simulator (LAMMPS) [28] with the Al$\mathrm{Mg}$ embedded atom method potential found in Ref. [29]. We calculated the distribution of dilute segregation energies using the large-cluster methodology outlined in Ref. [24], which allows us to account for the misorientation observed experimentally. Because of the weak interaction of $\mathrm{Mg}$ and vacancies [46,47], we do not expect nonequilibrium enrichment to play a significant role for $\mathrm{Mg}$.

Both facets of this $\Sigma 11$ GB have a variety of sites that are potential energy wells for $\mathrm{Mg}$ atoms to sit in (i.e., segregation energies $<0)$. Figure $1(\mathrm{~d})$ reveals that the $\{111\}$ boundary has a larger number of deeply attractive sites than the $\{200\}$ boundary. Applying the LangmuirMcLean segregation isotherm to this distribution of segregation energies $[32,48]$, our simulations predict that the $\{111\}$ facet will experience roughly double the $\mathrm{Mg}$ enrichment at room temperature compared to the $\{200\}$ facet -6.6 and $3.2 \mathrm{Mg}$ atoms $/ \mathrm{nm}^{2}$, respectively, under the approximation of noninteracting solutes. This segregation reduces the GB interfacial energy [49]. While the magnitude of reduction is calculated to be three times as strong for the $\{111\}$ facet as the $\{200\}$, the total interfacial energy of the $\{200\}$ remains lower (cf. Supplemental Material for numerical details [25]). The fine, periodic structuring of the boundary facets revealed by BF STEM can explain the periodic segregation observed by APT. Taken together with computational results showing facet-dependent drivers for segregation, these results suggest that the facet structure evolves self-consistently with solute segregation to minimize the overall free energy.

The cosegregation of $\mathrm{Mg}$ and $\mathrm{Zn}$ to $\mathrm{GBs}$ in $\mathrm{Al}-\mathrm{Zn}-\mathrm{Mg}$ $(\mathrm{Cu})$ alloys is frequently observed experimentally. These elements possess a strong tendency to precipitate as $\mathrm{Mg}-\mathrm{Zn}$ rich phase during aging [17], indicating an attractive interaction between $\mathrm{Mg}$ and $\mathrm{Zn}$. While we are unaware of a ternary $\mathrm{Al}-\mathrm{Zn}-\mathrm{Mg}$ empirical potential, two studies $[50,51]$ used density functional theory to study the same symmetric $\{120\}$ boundary. Our calculations show that $\mathrm{Mg}$ has a strong preference $(-0.30 \mathrm{eV})$ to bind to the planar site 

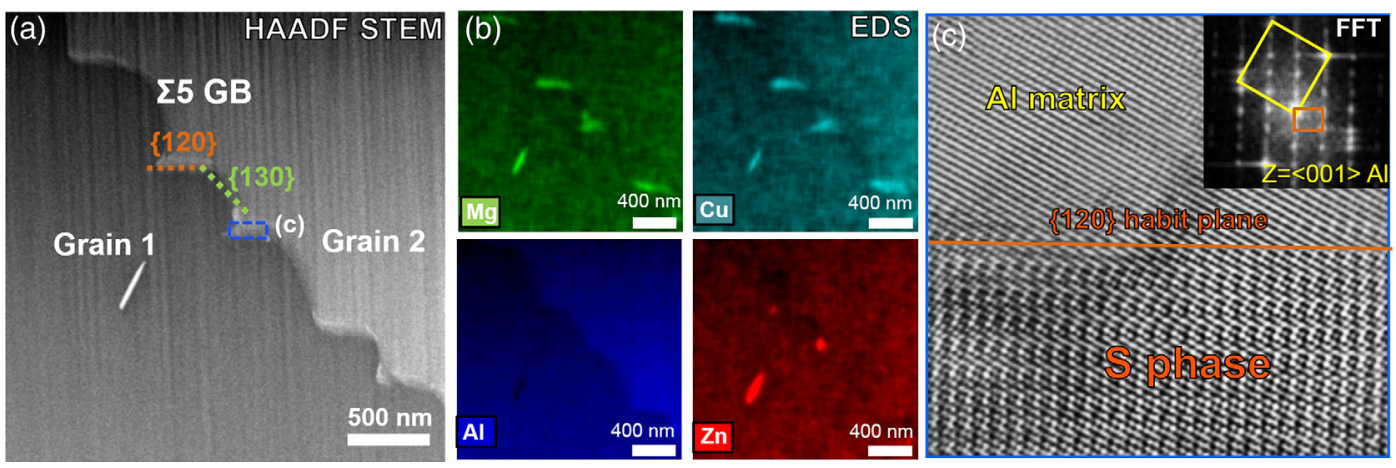

FIG. 2. (a) HAADF STEM image of a $\Sigma 5$ GB in the as-quenched alloy; (b) EDS maps of the faceted GB showing Mg-Cu enriched precipitates; (c) HAADF STEM image of the precipitate highlighted by blue rectangle in (a), with inset the corresponding Fast Fourier transform (FFT) pattern.

of this GB, while the density functional theory studies reported that $\mathrm{Zn}$ binds most strongly (approximately $-0.19 \mathrm{eV}$ ) to the nonplanar site closest to the plane. Not only do these solutes not compete for a preferred site, but since $\mathrm{Mg}$ and $\mathrm{Zn}$ prefer the expansive and compressive sites, respectively, the cosegregation observed experimentally may be explained by strain arguments.

Figure 2(a) illustrates the $\Sigma 5 \mathrm{~GB}$ in the as-quenched material characterized by $Z$-contrast high-angle annular dark-field images (HAADF) STEM performed along the common tilt $\langle 001\rangle_{\mathrm{Al}}$ zone axis. The average boundary plane is vicinal to the $\{180\}$ plane identified from the upper right grain. Here again, the GB contains two sets of alternating facets along the entire length, with each type highlighted by a colored line. The orange segments are symmetric $\{120\}$ facets, and the green segments are asymmetric $\{130\}$ plane. The average ratio of the $\{130\}$ facet lengths to the $\{120\}$ facet lengths is about 1.2. Figure 2(b) shows the energy dispersive spectroscopy analysis from the faceted $\Sigma 5 \mathrm{~GB}$. At this scale, we do not observe segregation at the $\{130\}$ facets, while the $\{120\}$ facets bear roughly $300 \mathrm{~nm}$ lathlike precipitates enriched with $\mathrm{Mg}$ and $\mathrm{Cu}$. The HAADF STEM image in Fig. 2(c) reveals that the precipitate has an orthorhombic structure, which matches the $S$ phase $\left(\mathrm{Al}_{2} \mathrm{CuMg}\right)$. It has an orientation relationship of $\langle 100\rangle_{s} / /\langle 100\rangle_{\mathrm{Al}}$, and an interface of $\{001\}_{S} / /\{120\}_{\mathrm{Al}}[52]$.

We performed complementary APT analyses on the same $\Sigma 5$ GB on another location, shown in Fig. 3. APT crystallography analysis [53] (Supplemental Material, Figs. 2 and 3 [25]) confirms that these results are consistent with our STEM observations. Two lathlike $S$ phases enriched in $\mathrm{Mg}$ and $\mathrm{Cu}$ are visible in Fig. 3(a), highlighted by isocomposition surfaces delineating regions containing
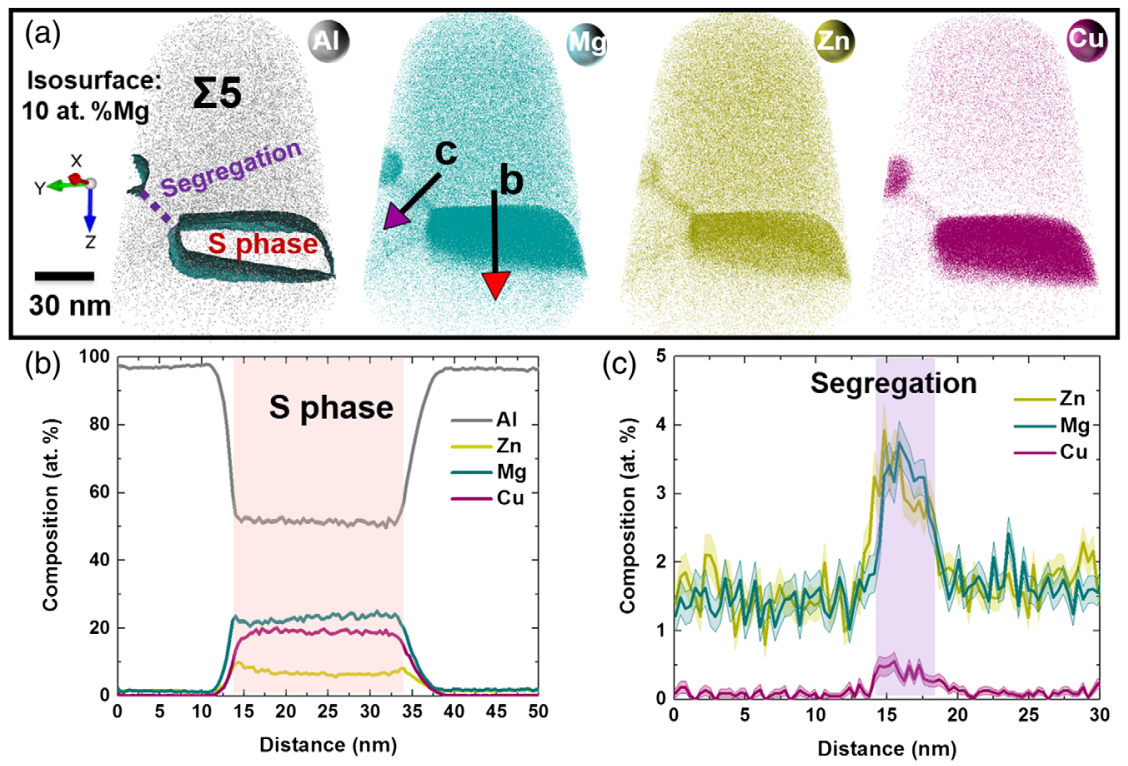

FIG. 3. APT analysis of the same $\Sigma 5$ GB with the STEM analysis: (a) Atom maps of all elements; (b) Corresponding composition profile across the precipitate calculated along the red arrow; (c) Corresponding composition profile across the region between two precipitates calculated along the purple arrow. 


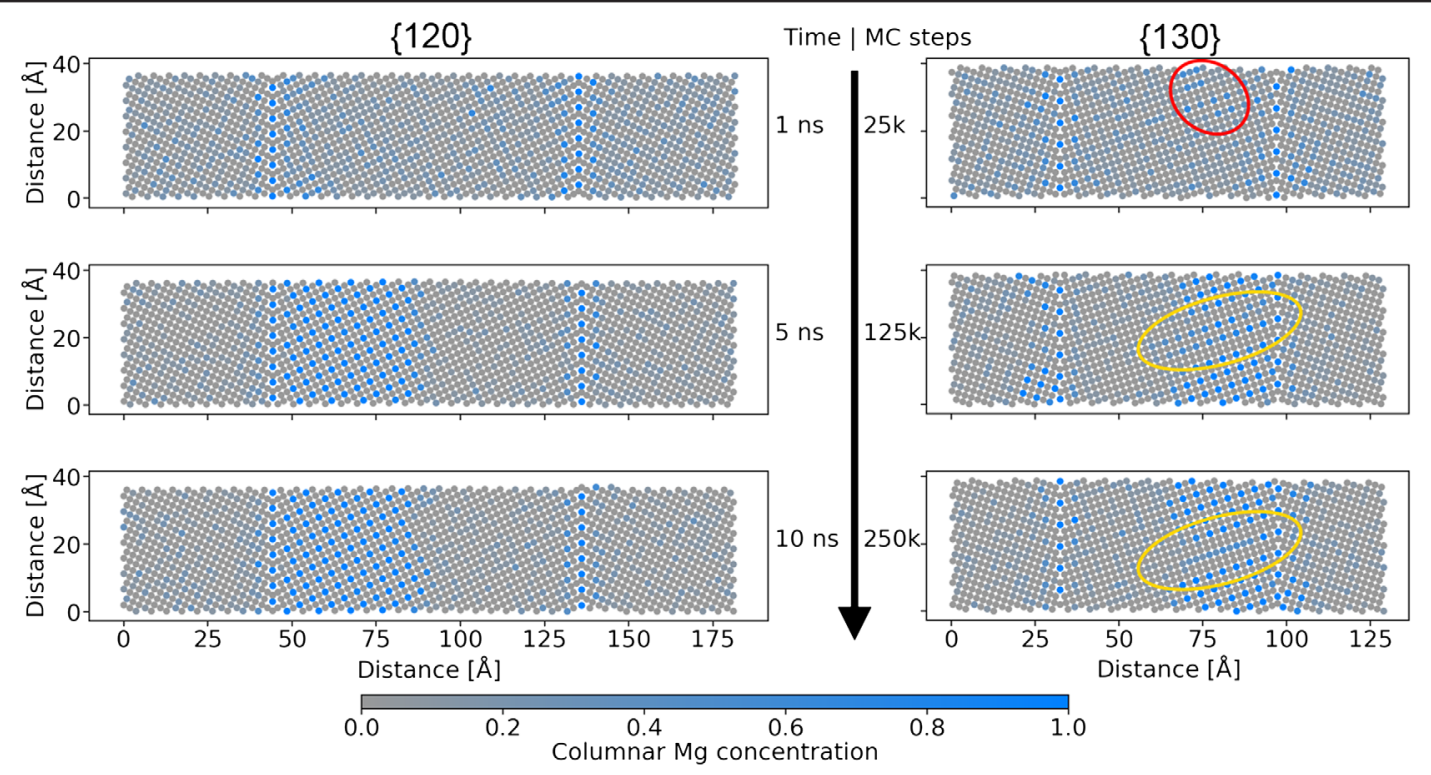

FIG. 4. VC SGC and MC-MD results for precipitate formation at $\{120\}$ and $\{130\}$ boundaries at $25^{\circ} \mathrm{C}$. Results are shown in an $\langle 001\rangle$ projection and colored according to $\mathrm{Mg}$ concentration. Snapshots are shown from three points in time for each boundary. Red and yellow ovals are discussed in the text.

more than 10 at. $\% \mathrm{Mg}$. A composition profile across the larger precipitate [Fig. 3(b)] shows average compositions of 52 at. $\% \mathrm{Al}, 23$ at. $\% \mathrm{Mg}, 18$ at. $\% \mathrm{Cu}$ and 7 at. $\% \mathrm{Zn}$ within the precipitate. This agrees with the stoichiometry of the $S$ phase $\left(\mathrm{Al}_{2} \mathrm{CuMg}\right)$ [54]. It is most likely that the 7 at. $\% \mathrm{Zn}$ substitutes to $\mathrm{Cu}$ sites, as $\mathrm{Cu}$ and $\mathrm{Zn}$ are both undersized, and the combined compositions of $\mathrm{Cu}(18$ at. \%) and $\mathrm{Zn}(7$ at. \%) equals 25 at. \%, as expected for the $\mathrm{Al}_{2} \mathrm{Mg}(\mathrm{Cu} / \mathrm{Zn})$ stoichiometry.

At this more detailed scale, we also observe $\mathrm{Zn}, \mathrm{Mg}$, and $\mathrm{Cu}$ enrichments along the $\{130\}$ facet that connects the two precipitates and the corresponding composition profile is plotted in Fig. 3(c). The average compositions in the enriched region are 3.1 at. $\% \mathrm{Zn}, 3.1$ at. $\% \mathrm{Mg}$, and 0.4 at. $\% \mathrm{Cu}$, which are enrichment factors of two in $\mathrm{Zn}$ and $\mathrm{Mg}$ and of three in $\mathrm{Cu}$ compared to the abutting bulk.

Both the $\{120\}$ and $\{130\}$ facets of this GB are thermodynamically favorable locations for $\mathrm{Zn}, \mathrm{Mg}$, and $\mathrm{Cu}$ segregation and even heterogeneous precipitation of the $S$ phase on the $\{120\}$ planes. Differential scanning calorimetry (Supplemental Material, Fig. 4 [25]) suggests that the observed $S$ phase formed during cooling from the solutionizing temperature of $475^{\circ} \mathrm{C}$. Previous research on highly concentrated Al-Zn-Mg-Cu alloys showed that the $S$ phase can form on GBs during quenching, and the precipitated volume increases with decreasing cooling rates [55-58]. Previous studies in $\mathrm{Al}-\mathrm{Cu}-\mathrm{Mg}$ alloys show that the $S$ phase forms laths on the $\{120\}_{\mathrm{Al}}$ planes, as its atomic arrangement in the $\{001\}$ planes is identical to that on the $\{120\}_{\mathrm{Al}}$ planes [52,59]. Hence, it is reasonable to consider that the $S$ phase preferentially nucleates at $\{120\}$ facets within the $\Sigma 5$ GBs, which ensures good coherency and low free energy of interfaces.
We then considered finite concentrations of solutes, i.e., beyond dilute segregation energies. We examined the formation of a Mg-rich phase near the symmetric $\{120\}$ and $\{130\} 36.87^{\circ}\langle 001\rangle \Sigma 5 \mathrm{GBs}$ by using the variance-constrained semi-grand-canonical (VC SGC) approach [37]. These coupled Monte Carlo-molecular dynamics (MC-MD) simulations were recently employed to study phase formation at dislocations in an Fe-based alloy [60]. At room temperature, the $\{120\}$ boundary has undergone a GB-phase transformation, similar to the transition observed by Frolov et al. [42], and we use this state as the starting point for further calculations (cf. Supplemental Material for details [25]). Figure 4 shows the evolution of both systems as a function of increasing MD time and MC steps, colored according to the columnar concentration of $\mathrm{Mg}$ along the misorientation axis (each column contains nine atoms). Both GBs experience various stages of segregation, from the initial decoration of the GB and adjacent planes, to the formation of $\mathrm{Al}_{3} \mathrm{Mg}$, the preferred precipitate for this $\mathrm{Al}-\mathrm{Mg}$ empirical potential.

Figure 4 reveals important differences between the formation of $\mathrm{Al}_{3} \mathrm{Mg}$ on the $\{120\}$ and $\{130\}$ planes. At the early stages, the $\{130\}$ system already shows hints of forming precipitates (circled red), opposite to the $\{120\}$. During growth, the $\{130\}$ system retains $\mathrm{Al}$ double-layer defects (circled yellow), even after $10 \mathrm{~ns}$ and numerous thousands of MC swaps. In contrast, the precipitate is undefected and coherent with the segregated $\{120\}$ plane.

To understand why this difference, we implemented a computational protocol using pyiron [30], which progressively includes new $\mathrm{Mg}$ atoms at the most energetically favorable site in our periodic computational domain 


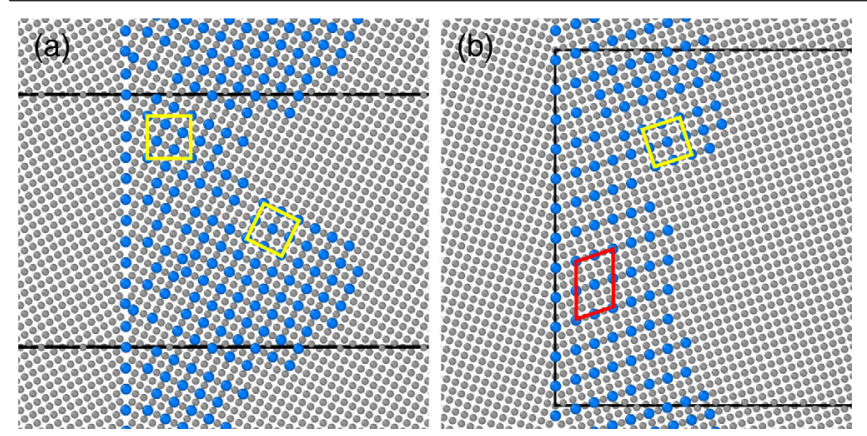

FIG. 5. Snapshots of Mg (large blue) segregation to the ideal $\Sigma 5$ symmetric: (a) $\{120\}$ and (b) $\{130\}$ boundaries in $\mathrm{Al}$ (small gray). Yellow squares indicate domains with an $\mathrm{Al}_{3} \mathrm{Mg}$ stoichiometry and similarly the red trapezoid indicates $\mathrm{Al}_{5} \mathrm{Mg}$. The black lines show periodic boundaries of the simulation domain (not all of which is shown).

(cf. Supplemental Material [25]). After the addition of many $\mathrm{Mg}$ atoms, in Fig. 5, the $\{120\}$ energy landscape encourages the coherent growth of $\mathrm{Al}_{3} \mathrm{Mg}$. To maintain a second-nearest-neighbor relationship between atoms segregated to the GB plane and additional atoms, the segregation at the $\{130\} \mathrm{GB}$ encourages the formation of $\mathrm{Al}_{5} \mathrm{Mg}$ exhibiting the Al-double layers seen in Fig. 4. Only farther from the $\{130\} \mathrm{GB}$ plane are new $\mathrm{Mg}$ atoms in the preferred $\mathrm{Al}_{3} \mathrm{Mg}$ configuration.

This model is simplified compared to the formation of the $S$ phase, which would require a quaternary $\mathrm{Al}-\mathrm{Zn}-\mathrm{Mg}-$ $\mathrm{Cu}$ potential. Nonetheless, our calculations demonstrated the influence of GB geometry in the formation and growth of precipitates. In this case, the underlying $0 \mathrm{~K}$ energetic preference of the $\{130\}$ boundary for a structure with $\mathrm{Al}$ double layers persists even at elevated temperatures, perturbing the formation of $\mathrm{Al}_{3} \mathrm{Mg}$. Similarly, we suggest that the geometry and segregation pattern of the $\{120\}$ boundary forms a preferable "epitaxial substrate" for growing the $S$ phase compared to other boundaries.

We demonstrate that GBs in an engineering $\mathrm{Al}$ alloy can be composed of solute or precipitate-rich and solute or precipitate-depleted facets. The simulations show that the periodic segregation and preferential precipitation have a strong dependence on the GB planes. Hsieh and Balluffi observed that the $\Sigma 3$ and $\Sigma 11$ GBs dissociate into a series of facets along lower energy planes in pure-Al bicrystals assisted by thermally induced fluctuations [5]. However, segregation can also reduce the interfacial energy and promote anisotropy and faceting as segregated solutes influence GB stress due to the misfit between solutes and solvent. Previous studies have shown that segregation of $\mathrm{Bi}$ causes $\mathrm{GB}$ faceting in $\mathrm{Cu}$ [61-65]. Direct atomic-resolution STEM investigation also demonstrates that the segregation of Ag induces nanofaceting transition in a $\mathrm{Cu}$ bicrystal [11]. In light of our findings, the faceting and segregation likely take place during the homogenization of the alloy and these two energy-reduction mechanisms are strongly coupled $[9,66]$.
The observed $\Sigma 5$ GB composes of $\{120\}$ and $\{130\}$ type low-energy planes and the $\Sigma 11$ GB contains $\{200\}$ and $\{111\}$ type low-index facets. The periodic segregation results from preferential segregation to particular facets, which themselves develop to minimize their interfacial energy by dissociating into a series of low-energy facets-including segregation effects. The asymmetric segregation strongly changes the excess volume and stress of GBs, which promotes stronger anisotropy and consequently affects the interaction between the different facets and controls the shape and size of the faceted GBs. In such a precipitation-hardened alloy, we further expect that the details of segregation will influence the formation of precipitates in the localized segregated region, as exemplified with the observation of the $S$ phase.

To conclude, in an engineering $\mathrm{Al}-\mathrm{Zn}-\mathrm{Mg}-\mathrm{Cu}$ alloy, we investigated in detail the structure and composition of a $\Sigma 5$ and a $\Sigma 11$, as well as a $\Sigma 13 a$ GB only by APT. We showed evidence of a complex interplay between solute segregation and faceting of the GBs. Although the character of individual GB is often overlooked in the segregation study in engineering $\mathrm{Al}$ alloys, our study enriches the current understanding of the chemical-structural interactions. The solute segregation and heterogeneous precipitation at GBs have an impact on the corrosion properties of $\mathrm{Al}$ alloys $[19,67]$. Within the same GB, the facets coupled with the anisotropic segregation and precipitation behavior will significantly influence the GB cohesion, intergranular fracture, and corrosion resistance of engineering $\mathrm{Al}$ alloys. Better predictions for the temporal evolution of the alloy's microstructure during aging and in service will require knowledge of the solute distribution within the microstructure, particularly at GBs.

H. Z. acknowledges support from the China Scholarship Council (CSC). G. D. acknowledges funding by the European Research Council (Grant No. 787446-GB-CORRELATE).

*Corresponding author.

h.zhao@mpie.de

Corresponding author.

w.lu@mpie.de

Corresponding author.

b.gault@mpie.de

[1] M. P. Harmer, Science 332, 182 (2011).

[2] P. R. Cantwell, M. Tang, S. J. Dillon, J. Luo, G. S. Rohrer, and M. P. Harmer, Acta Mater. 62, 1 (2014).

[3] D. Raabe, M. Herbig, S. Sandlöbes, Y. Li, D. Tytko, M. Kuzmina, D. Ponge, and P. P. Choi, Curr. Opin. Solid State Mater. Sci. 18, 253 (2014).

[4] J. Luo, H. Cheng, K. M. Asl, C. J. Kiely, and M. P. Harmer, Science 333, 1730 (2011).

[5] T. Hsieh and R. Balluffi, Acta Metall. 37, 2133 (1989).

[6] H. Sternlicht, W. Rheinheimer, M. J. Hoffmann, and W. D. Kaplan, J. Mater. Sci. 51, 467 (2016).

[7] R. Balluffi, Metall. Mater. Trans. B 13, 527 (1982). 
[8] A. Kwiatkowski da Silva, D. Ponge, Z. Peng, G. Inden, Y. Lu, A. Breen, B. Gault, and D. Raabe, Nat. Commun. 9, 1137 (2018).

[9] Z. Yu, P. R. Cantwell, Q. Gao, D. Yin, Y. Zhang, N. Zhou, G. S. Rohrer, M. Widom, J. Luo, and M. P. Harmer, Science 358, 97 (2017).

[10] C. H. Liebscher, A. Stoffers, M. Alam, L. Lymperakis, O. Cojocaru-Mirédin, B. Gault, J. Neugebauer, G. Dehm, C. Scheu, and D. Raabe, Phys. Rev. Lett. 121, 015702 (2018).

[11] N. J. Peter, T. Frolov, M. J. Duarte, R. Hadian, C. Ophus, C. Kirchlechner, C. H. Liebscher, and G. Dehm, Phys. Rev. Lett. 121, 255502 (2018).

[12] D. L. Medlin, K. Hattar, J. A. Zimmerman, F. Abdeljawad, and S. M. Foiles, Acta Mater. 124, 383 (2017).

[13] B. Straumal, S. Polyakov, E. Bischoff, W. Gust, and E. Mittemeijer, Interface Sci. 9, 287 (2001).

[14] W. R. Wagner, T. Tan, and R. Balluffi, Philos. Mag. 29, 895 (1974).

[15] J. C. Hamilton, D. J. Siegel, I. Daruka, and F. Leonard, Phys. Rev. Lett. 90, 246102 (2003).

[16] X. M. Li and M. J. Starink, J. Alloys Compd. 509, 471 (2011).

[17] H. Zhao, F. De Geuser, A. Kwiatkowski da Silva, A. Szczepaniak, B. Gault, D. Ponge, and D. Raabe, Acta Mater. 156, 318 (2018).

[18] D. Vaughan, Acta Metall. 18, 183 (1970).

[19] R. G. Song, W. Dietzel, B. J. Zhang, W. J. Liu, M. K. Tseng, and A. Atrens, Acta Mater. 52, 4727 (2004).

[20] B. Blum, M. Menyhard, D. E. Luzzi, and C. J. McMahon, Scr. Metall. Mater. 24, 2169 (1990).

[21] A. Donald, Philos. Mag. 34, 1185 (1976).

[22] B. Straumal, S. Polyakov, and E. Mittemeijer, Acta Mater. 54, 167 (2006).

[23] S. B. Lee, W. Sigle, and M. Rühle, Acta Mater. 51, 4583 (2003).

[24] Z. Wang, M. Saito, K. P. McKenna, L. Gu, S. Tsukimoto, A. L. Shluger, and Y. Ikuhara, Nature (London) 479, 380 (2011).

[25] See Supplemental Material at http://link.aps.org/supplemental/ 10.1103/PhysRevLett.124.106102 for the experimental and simulation details, which includes Refs. [17,26-45].

[26] H. Zhao, B. Gault, D. Ponge, D. Raabe, and F. De Geuser, Scr. Mater. 154, 106 (2018).

[27] B. Gault, M. P. Moody, F. de Geuser, G. Tsafnat, A. La Fontaine, L. T. Stephenson, D. Haley, and S. P. Ringer, J. Appl. Phys. 105, 034913 (2009).

[28] S. Plimpton, J. Comput. Phys. 117, 1 (1995).

[29] M. Mendelev, M. Asta, M. Rahman, and J. Hoyt, Philos. Mag. 89, 3269 (2009).

[30] J. Janssen, S. Surendralal, Y. Lysogorskiy, M. Todorova, T. Hickel, R. Drautz, and J. Neugebauer, Comput. Mater. Sci. 163, 24 (2019).

[31] R. Hadian, B. Grabowski, and J. Neugebauer, J. Open Source Softw. 3, 900 (2018).

[32] L. Huber, R. Hadian, B. Grabowski, and J. Neugebauer, npj Comput. Mater. 4, 64 (2018).

[33] https://github.com/liamhuber/clustergb.

[34] B.-J. Lee and S.-H. Choi, Model. Simul. Mater. Sci. Eng. 12, 621 (2004).

[35] J. D. Honeycutt and H. C. Andersen, J. Phys. Chem. 91, 4950 (1987).
[36] D. Faken and H. Jónsson, Comput. Mater. Sci. 2, 279 (1994).

[37] B. Sadigh, P. Erhart, A. Stukowski, A. Caro, E. Martinez, and L. Zepeda-Ruiz, Phys. Rev. B 85, 184203 (2012).

[38] https://vcsgc-lammps.materialsmodeling.org.

[39] M. Ester, H.-P. Kriegel, J. Sander, and X. Xu, in Proceedings of the 2nd International Conference on Knowledge Discovery and Data Mining (AAAI Press, Portland, OR, 1996), p. 226.

[40] E. Schubert, J. Sander, M. Ester, H. P. Kriegel, and X. Xu, ACM Trans. Database Syst. (TODS) 42, 19 (2017).

[41] F. Pedregosa et al., J. Mach. Learn. Res. 12, 2825 (2011).

[42] T. Frolov, M. Asta, and Y. Mishin, Phys. Rev. B 92, 020103 (R) (2015).

[43] A. Stukowski, Model. Simul. Mater. Sci. Eng. 18, 015012 (2010).

[44] A. Stukowski, Model. Simul. Mater. Sci. Eng. 20, 045021 (2012).

[45] X.-Y. Liu, P. Ohotnicky, J. Adams, C. L. Rohrer, and R. Hyland, Jr., Surf. Sci. 373, 357 (1997).

[46] M. Mantina, Y. Wang, L. Chen, Z. Liu, and C. Wolverton, Acta Mater. 57, 4102 (2009).

[47] R. W. Balluffi and P. S. Ho, Diffusion (American Society for Metals, Metals Park, Ohio, 1973), p. 83.

[48] C. White and W. Coghlan, Metall. Mater. Trans. A 8, 1403 (1977).

[49] R. Kirchheim, Acta Mater. 55, 5129 (2007).

[50] S. Zhang, O. Y. Kontsevoi, A. J. Freeman, and G. B. Olson, Acta Mater. 59, 6155 (2011).

[51] M. Petrik, A. Kuznetsov, N. Enikeev, Y. N. Gornostyrev, and R. Valiev, Phys. Met. Metallogr. 119, 607 (2018).

[52] V. Radmilovic, R. Kilaas, U. Dahmen, and G. Shiflet, Acta Mater. 47, 3987 (1999).

[53] B. Gault, M. P. Moody, J. M. Cairney, and S. P. Ringer, Mater. Today 15, 378 (2012).

[54] M. J. Styles, R. K. W. Marceau, T. J. Bastow, H. E. A. Brand, M. A. Gibson, and C. R. Hutchinson, Acta Mater. 98, 64 (2015).

[55] Y. Zhang, B. Milkereit, O. Kessler, C. Schick, and P. A. Rometsch, J. Alloys Compd. 584, 581 (2014).

[56] B. Yang, B. Milkereit, Y. Zhang, P. A. Rometsch, O. Kessler, and C. Schick, Mater. Charact. 120, 30 (2016).

[57] D. Godard, P. Archambault, E. Aeby-Gautier, and G. Lapasset, Acta Mater. 50, 2319 (2002).

[58] C. Mondal and A. K. Mukhopadhyay, Mater. Sci. Eng. A 391, 367 (2005).

[59] G. B. Winkelman, K. Raviprasad, and B. C. Muddle, Acta Mater. 55, 3213 (2007).

[60] V. Turlo and T. J. Rupert, Phys. Rev. Lett. 122, 126102 (2019).

[61] T. G. Ference and R. W. Balluffi, Scr. Metall. 22, 1929 (1988).

[62] A. M. Donald and L. M. Brown, Acta Metall. 27, 59 (1979).

[63] W. Sigle, L. S. Ciiang, and W. Gusr, Philos. Mag. A 82, 1595 (2002).

[64] G. Duscher, M. F. Chisholm, U. Alber, and M. Rühle, Nat. Mater. 3, 621 (2004).

[65] U. Alber, H. Müllejans, and M. Rühle, Acta Mater. 47, 4047 (1999).

[66] E. D. Hondros, J. Phys. Colloques 36, C4 (1975).

[67] L. Christodoulou and H. M. Flower, Acta Metall. 28, 481 (1980). 